\title{
AUTOMATION AND ROBOTIZATION OF MOUNTING OPERATIONS IN BUILDING
}

\author{
Thomas Bock*, Dmitrij Parschin**, Alexej Bulgakow* \\ *Technical University Munich,Thomas.Bock@lrz.tu-muenchen.de \\ ** Rostov State Building University, RUSSIA, pragma_plus@mail.ru
}

\begin{abstract}
Construction is of great interest for applying practical and theoretical results in the field of robotics. The Economic efficiency potential of using robots is defined by construction quantities, high level of manual work, hard and unfavorable working conditions. Mounting operations are the main focus for the practical use of robots. The lecture describes technological characteristics features of such operations, kinematic structures for mounting operations are suggested and kinematic models design are shown as an example. Taking into consideration management details of mounting operations the recommendations on movements planning and forming the laws of drives control are given. In conclusion, the recommendations for applying the results and using computer technologies of developing control programs are presented.
\end{abstract}

Key words: robots, construction, building, kinematics, control.

\section{INTRODUCTION}

Mechanization and automation of construction operations through conventional methods not always allow one to meet the goal. The introduction of robots promotes changes of construction technology and brings it on a new level. The analysis of the main types of construction work shows that mounting operations are of the greatest practical interest. On the basis of the technological and economical analysis of this work the preferable technological operations being subject to robotization are defined. Construction mounting operations include mounting of components and structures, welding during mounting [1,2]. The analysis of developments in the field of construction robotics shows that a positive effect can be only achieved creating special-purpose robots taking into account technological, design and operational features of construction operations to the full extent. The robotization of mounting operations is connected with the provision of the preset of orientation and installation of components and structures. The components to be mounted howing large overall sizes and weights are distinguished by relationship of overall sizes and manner of fixation in the design position. The development of mounting robots and robotized mounting technology is connected with settling difficult tasks in designing kinematic structures, mathematical and algorithmic provision.

In view of the described features of robotizing mounting operations let us consider the principles of kinematic structures design and tackling the problems of planning movements and end-effectors control in mounting robots.

\section{KINEMATICS STRUCTURE OF MOUNTING ROBOTS}

The analysis of mounting technology and the stated requirements show that the successful solving of robotization tasks is defined by the development of original kinematics structures and by the structural synthesis fulfilled in a proper way. To fulfil different mounting operations robotic dedicated means are necessary which have such features as load capacity, service range and functions to be carried out. Depending on the type of functions to be performed it may be recommended three main types of kinematics structures for mounting robots: construction-mounting robots equipped with a telescopic arm placed on a tool carrier; tower crane-manipulators travelling on a rail track; mobile orienting robots.

For construction-mounting robots of the first type a structural design with 6 degrees of freedom is suggested which is presented in fig.1. The structural analysis of this design has shown that it provides handling, installation and orientation of components at a using activity with height up to $10-15 \mathrm{~m}$ with minimum costs of power and time resources. As the basic type of such robots may be taken a mobile crane with telescopic arm an additional turning boom with orienting mobile and gripping automatic traverse being installed on it. The main purpose of these robots is mounting of cottages, administration buildings, industrial objects and different pavilions. 
The structure of the translational degrees of freedom provides components handling to the place of their mounting with regard of limitation on the trajectory of its movement. Vertical design is made available by the position of the boom 4 but the orientation

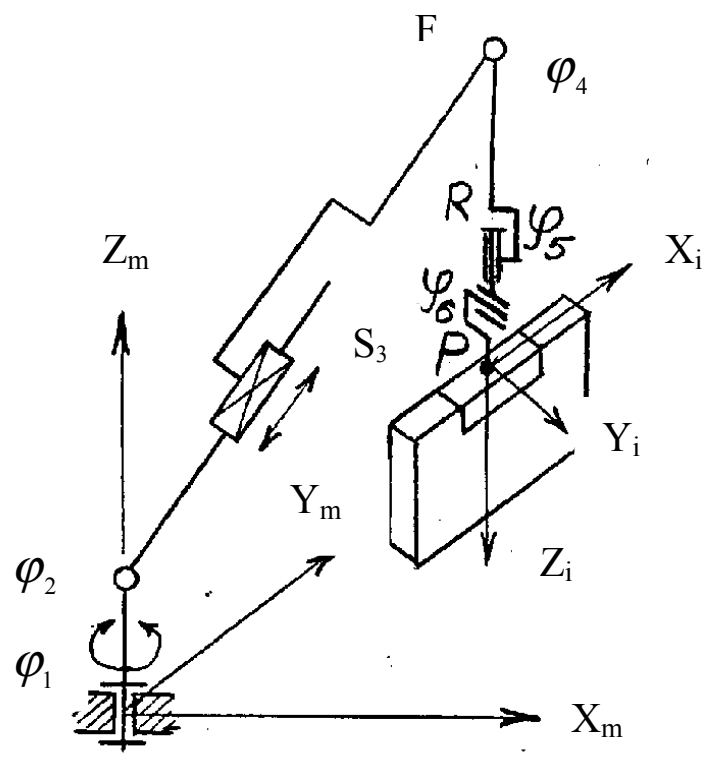

Figure 1. Construction-mountig robot with 6 degrees of freedom

around the vertical axis $Z i$ and avoidance of pitch relative to axis $X i$ is made by degrees of freedom of the orienting module.

While perfoming mounting operations at the using activities with height up to $10-25 \mathrm{~m}$ the tower cranes-manipulators has been found advantageous (fig.2). The tower with the arm installed on the

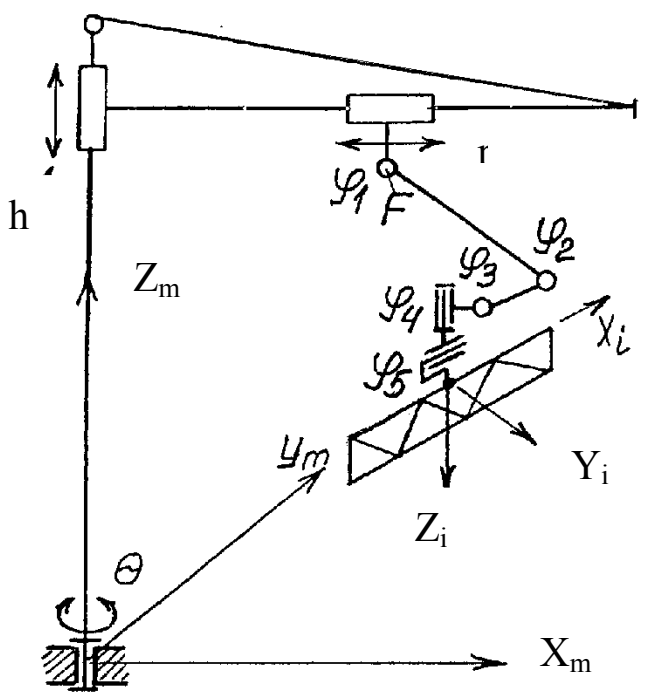

Figure 2. The tower crane-manipulator

movable truck have three degrees of freedom: turn $\theta$, the arm hoisting $h$ and the truck moving along the arm $r$. The manipulator with two turning links and the orienting module with three degrees of freedom are installed on the trucks. In case of fulfilling mounting operations under the conditions when there is a small area it is expedient that the orienting module to be installed on a horizontal traverse having flexible and rigid connection with the arm and the tower. One of the applications of such cranes-manipulators is supposed to be the mounting of prefabricated multistory residential buildings. Robots performed by the authors show the possibility of programming control over the whole process subject to the condition that the automatic system of control over the displacement and orientation of the units to be mounted is used.

When mounting high-rise buildings one of the promising trends should be considered the develoment of robotized mounting systems, which are made op, the basis of tower cranes with program control and a mounting robot (fig.3). The crane

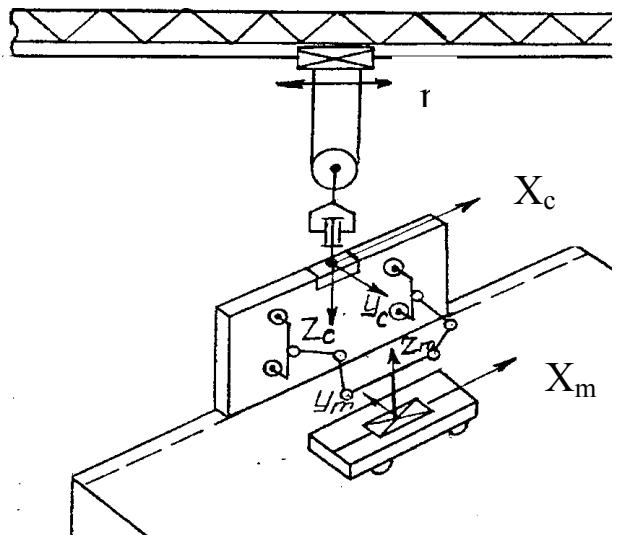

Figure 3. The robotized mounting systems

provides travelling of the unit the mounting area, its previous installation and keeping it during the process of orientation. The mounting robot provides the position correction and the precision orientation of the component while mounting. The structural analysis has shown that it is expedient to use a twoarm manipulation installed on a self-propelled platform and having in its base a transnational degree of freedom. The platform is supplied with retracting controlled supports. The position control over the component being mounted is performed by the laser orientation devices that allows to provide continuous control and auto mate the process of mounting.

Control of mounting robots can be performed either in the coordinate system of the manipulator $X_{m} Y_{m} Z_{m}$ or in the coordinate system of the building $X_{b} Y_{b} Z_{b}$. It is recommended to plan transport movements $\mathrm{n}$ the system $X_{m} Y_{m} Z_{m}$ and adjusting, orienting and technological operations to perform in the coordinate system. $X_{b} Y_{b} Z_{b}$. The position of coordinate systems while developing kinematic model must ensure conventional representation of mechanisms locations and simplify the process of 
coordinates transformation. For the manipulator under consideration the basic coordinate system $X_{m} Y_{m} Z_{m}$ is connected with the rotation axis and the axis $X_{m}$ is directed along the self-propelled truck. For the rotational degrees of freedom, axes of which are perpendicular to the links $\left(2^{\text {nd }}, 4^{\text {th }}, 6^{\text {th }}\right.$ pairs $)$ it is convenient to combine axes $X$ with rotation axes and axes $Z$ to be led along the link. For the translational degree of freedom and the rotational one with the location of the axis along the lint it is necessary to direct axes $Z$ along the axes of movement and axes $X$ to combine with axes $X$ of the previous degrees of freedom. It is convenient to direct axis $Z_{i}$ of the grasp coordinate system along the last link and axis $X_{i}$ - along the traverse of the gripping device. In this case the position and orientation of point $F$ (fig.1) with help of which we should set transport paths of the movement, are described by the matrix:

$$
T_{F}=T_{1} T_{2} T_{3}=\left[\begin{array}{cccc}
C \varphi_{1} & -S \varphi_{1} C \varphi_{2} & S \varphi_{1} S \varphi_{2} & S_{3} S \varphi_{1} S \varphi_{2} \\
S \varphi_{1} & C \varphi_{1} C \varphi_{2} & -C \varphi_{1} S \varphi_{2} & -S_{3} C \varphi_{1} S \varphi_{2} \\
0 & S \varphi_{2} & C \varphi_{2} & l_{1}+S_{3} C \varphi_{2} \\
0 & 0 & 0 & 1
\end{array}\right]
$$

The configuration of the kinematic model ought to comprise the description of position and location of point $R$ defining the initial position of the orienting module:

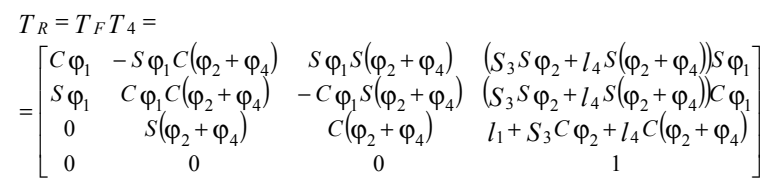
The change of the component position and orientation during the operationof the orienting degrees of freedom is described by the transformation matrix:

$$
T_{o r}=T_{5} \cdot T_{6}=\left[\begin{array}{cccc}
-S \varphi_{5} \cdot C \varphi_{6} & -C \varphi_{5} & S \varphi_{5} \cdot S \varphi_{6} & l_{6} \cdot S \varphi_{5} \cdot S \varphi_{6} \\
C \varphi_{5} \cdot C \varphi_{6} & -S \varphi_{5} & -S \varphi_{6} \cdot C \varphi_{5} & -l_{6} \cdot C \varphi_{5} \cdot S \varphi_{6} \\
S \varphi_{6} & 0 & C_{6} & l_{1}+l_{6} \cdot C \varphi_{6} \\
0 & 0 & 0 & 1
\end{array}\right]
$$

Using these matrix we can define the position and orientation of the component being mounted with help of transformation matrix: $T_{P}=T_{F}+T_{o r}$. On the basis of this matrix we get the system of equations to determine coordinates of point $P$ in the coordinate system of the robot $X_{m} Y_{m} Z_{m}$ :

$$
\begin{aligned}
& X_{p}=l_{6} \cdot C \varphi_{1} S \varphi_{5} S \varphi_{6}+l_{6} \cdot S \varphi_{1} C\left(\varphi_{2}+\varphi_{4}\right) C \varphi_{5} S \varphi_{6}+ \\
& +\left(l_{5}+l_{6} C \varphi_{6}\right) S \varphi_{1} S\left(\varphi_{2}+\varphi_{4}\right)+\left(S_{3} S \varphi_{2}+l_{4} S\left(\varphi_{2}+\varphi_{4}\right)\right) S \varphi_{1} ; \\
& Y_{p}=l_{6} \cdot S \varphi_{1} S \varphi_{5} S \varphi_{6}+l_{6} \cdot C \varphi_{1} C\left(\varphi_{2}+\varphi_{4}\right) C \varphi_{5} S \varphi_{6}+ \\
& +\left(l_{5}+l_{6} C \varphi_{6}\right) C \varphi_{1} S\left(\varphi_{2}+\varphi_{4}\right)+\left(S_{3} S \varphi_{2}+l_{4} S\left(\varphi_{2}+\varphi_{4}\right)\right) C \varphi_{1} \\
& Z=l_{1}+S_{3} C \varphi_{2}+l_{4} \cdot C\left(\varphi_{2}+\varphi_{4}\right)+\left(l_{5}+l_{6} C \varphi_{6}\right) C\left(\varphi_{2}+\varphi_{4}\right)+ \\
& +l_{6} C \varphi_{5} S \varphi_{6} S\left(\varphi_{2}+\varphi_{4}\right) .
\end{aligned}
$$

When controlling the robot's movement the data about the speeds of the grip motions and the point defining the transport path of the movement are necessary. At the given speeds of the manipulator links motions the views of the gripper linear speed on the axis $X_{m} Y_{m} Z_{m}$ are defined by the equations: The speeds of the arm upper point ( point $F$ ) motion are described by the equations:

$$
\begin{aligned}
& v_{x}^{(F)}=S_{3}^{\prime} \cdot S \varphi_{1} S \varphi_{2}+S_{3} C \varphi_{1} S \varphi_{2} \cdot \varphi_{1}^{\prime}+S_{3} S \varphi_{1} C \varphi_{2} \cdot \varphi_{2}^{\prime} ; \\
& v_{y}^{(F)}=-S_{3}^{\prime} \cdot C \varphi_{1} S \varphi_{2}+S_{3} S \varphi_{1} S \varphi_{2} \cdot \varphi_{1}^{\prime}-S_{3} C \varphi_{1} C \varphi_{2} \cdot \varphi_{2}^{\prime} ; \\
& v_{z}^{(F)}=S_{3}^{\prime} \cdot C \varphi_{2}+S_{3} S \varphi_{2} \cdot \varphi_{2}^{\prime} .
\end{aligned}
$$

Kinematic models of mounting robots must include the systems of equations to define positions of the degrees of freedom and speeds of their movements, ensuring the preset grasp position and the required speed of its movement. These equations are derived on the basis of the transformation matrix elements $T_{F}, T_{R}$ and $T_{o r}$ by the way of simultaneous solution of the system of equations and heir multiple transformations. To use the methods of successive approximations for solving inverse kinematic problems is not recommended. To simplify the development of kinematic models we suggest to use the method of decomposition of the structure, the essence of which is in breaking the architecture into elementary type structures. This allows to have transformation matrix for each type module in advance that connect its input and output coordinate systems.

\section{MOTION PLANNING OF MOUNTING ROBOTS}

Mounting robots' motions planning should be performed in the form of setting motion and velocity vectors in projections on the axis of the robot coordinate system:

$$
\begin{aligned}
& X_{P}{ }^{(t)}=\left[x_{p}{ }^{(t)}, y_{p}{ }^{(t)}, z_{p}{ }^{(t)}\right] ; \\
& V_{P}(t)=\left[\boldsymbol{V}_{x p}{ }^{(t)}, \boldsymbol{V}_{y p}{ }^{(t)}, \boldsymbol{V}_{z p}(t)\right]
\end{aligned}
$$

When performing most technological operations by construction robots velocity module is chosen constant.

Motions planning of mounting robots first of all includes design of a component trajectory to handle if to the mounting area. The analysis of the mounting technology has show that it is most favorable to use trajectories consisting of two or three rectilinear sections. They are sure to include vertical hoisting of the component to the preset height and its horizontal displacement along the straight line to the point of positioning. At high and extended object components handling can be carried out along the vertical hoist of the component from the cassette. The vertical motion is described by the equations:

$$
X_{1}(t)=\left[x_{0}, y_{0}, z_{0}+v_{z} t\right] ; \quad V_{P}=\left[o, o, v_{z}\right]
$$

While carrying out this motion the vertical position of the component must be kept. The characteristic 
feature of mounting robots' motions planning is defining the degrees of freedom, ensuring handling operations and forming control laws for them. Incase of the robot with kinematics structure presented in fig.1 the performance of vertical hoisting the component with its position being unaltered is provided by the degrees of freedom $\varphi_{2}, S_{3}, \varphi_{4}$ :

$$
\begin{aligned}
& \varphi_{2}(t)=v_{z}{ }^{t} / r_{F} ; \\
& S_{3}(t)=\left(v_{z}^{2}+r_{F}^{2}\right)^{1 / 2} ; \\
& \varphi_{4}(t)=-\left(\pi+\varphi_{2}(t)\right),
\end{aligned}
$$

where $r_{F}^{2}=x_{F}^{2}+y_{F}^{2}$.

The horizontal displacement of the component from point $H\left(x_{h}, y_{h}, z_{h}\right)$ to the point $P\left(x_{p}, y_{p}, z_{p}\right)$ of positioning is described by the vector

$$
X_{2}(t)=\left\lfloor\left(x_{h}+v_{x} t\right),\left(y_{h}+v_{y} t\right), z_{h}\right\rfloor
$$

and the components of the velocity vector are equal to

$$
\begin{aligned}
& v_{x}=|v| \cdot\left(x_{p}-x_{h}\right) / l_{P h} ; \\
& v_{x}=|v| \cdot\left(y_{p}-y_{h}\right) / l_{P h} ; \\
& v_{x}=0 \\
& \text { were } l_{P h}=\left(\left(x_{p}+x_{h}\right)^{2}+\left(y_{p}+y_{h}\right)^{2}\right)^{1 / 2} .
\end{aligned}
$$

For the robot under consideration such motions are provided by the degrees of freedom $\mathrm{Y}_{1}, \mathrm{Y}_{2}, \mathrm{Y}_{3}, \mathrm{Y}_{4}$ are controlled according to the expressions:

$$
\begin{aligned}
& \varphi_{1}(t)=\operatorname{arctg}\left[\left(y_{h}+v_{y} t\right) /\left(x_{h}+v_{x} t\right)\right] ; \\
& \varphi_{2}(t)=\operatorname{arctg}\left[\left(z_{p}-l_{1}\right) / l(t)\right] ; \\
& \varphi_{4}(t)=\varphi_{2}(t)
\end{aligned}
$$

where $l(t)=\left(x_{h}+v_{x} t\right)^{2}+\left(y_{h}+v_{y} t\right)^{2}$.

Links drives control in the regions of the component hoisting and displacement to the region of positioning should be carried out along the velocity vector under position control. During this operation we recommend to start with constant acceleration and to retard when coming to the point of positioning according to the law:

$$
v(t)=v_{n} \exp (-k t) ; w(t)=w_{\max } \exp (-k t),
$$

where $k$ - the coefficient of braking intensity.

The component being displaced to the point of positioning, the process of its orientation takes place so that the gripping device coordinate system $X_{i} Y_{i} Z_{i}$ becomes coaxial with the coordinate system $X_{b} Y_{b} Z_{b}$ of the installation. Orienting degrees control should be carried out on the basis of the synthesized control laws taking into account inertia characteristics of the component, manipulator and drive links. The most critical and highly precision operation is a component fit after its orientation that requires axis timing. When developing algorithms the manipulator is to be viewed as a multilane mechanism with dynamically connected movements of separate links. The dynamics of the degrees of freedom can be decomposed and the amount of calculations can be reduced by the way of introducing adaptive control of robot's actuating mechanisms on the basis of the system decomposition. In this case control is generated in the form of two components:

$$
\bar{U}=\bar{U}_{p}+\bar{U}_{A} \text {. }
$$

Program control $\left(\bar{U}_{P}\right)$ is synthesized with account of the guide dynamics and the adaptive component $\left(\bar{U}_{A}\right)$ with adjustable parameters is determined by the expression:

$$
\bar{U}_{A}=L \cdot \bar{X}+K \cdot \bar{U} ;
$$

where $L$ is the matrix of adjustable parameters including dynamics and utual influence of channels; $K$ is the matrix of parameters including decomposition of controlling signals; $X$ is the vector of state parameters.

\section{CONCLUSION}

The presented material is prepared on the basis of the authors' research which was carried out while developing proposals on automation and robotization of operations in residential and industrial construction. Kinematics structures and recommendations on motions planning for mounting robots proposed here can be used while developing designs for robotization of construction work. On their basis we can obtain different robots' design solutions. While developing kinematics models we should combine matrix, vector and geometry methods for solving problems of kinematics with the purpose of getting simpler expressions, reducing the amount of calculations. Computer simulation of motions of mounting robots has shown the efficiency of the described methods of planning motions trajectories and drives control. Therefore the presented approaches can serve as recommendations while developing control algorithms for mounting robots.

\section{REFERENCES}

[1] V. Zagorodnjuc, D.Parchin., The Construction Rootica (Mockow, 1990).

[2] T.Furukawa and S. Kikukawa. "Introduction of Robots to the Construction Work Site". Robot, No. 38 (1983). 